\title{
Chemical Characterization and Bioactive Properties of Different Extracts from Fibigia clypeata, an Unexplored Plant Food
}

\author{
Gokhan Zengin $1, * \mathbb{C}$, Mohamad Fawzi Mahomoodally ${ }^{2,3}$, Gabriele Rocchetti ${ }^{4}{ }^{\mathbb{D}}$, \\ Luigi Lucini $^{4}\left(\mathbb{D}\right.$, Elwira Sieniawska ${ }^{5}$ (D) , tukasz Świątek ${ }^{6}$, Barbara Rajtar ${ }^{6}$ (D), \\ Małgorzata Polz-Dacewicz ${ }^{6}$, Ismail Senkardes ${ }^{7}$ (D), Abdurrahman Aktumsek ${ }^{1}$, \\ Marie Carene Nancy Picot-Allain ${ }^{3}$ (D) and Domenico Montesano ${ }^{8, *(D)}$ \\ 1 Department of Biology, Science Faculty, Selcuk University, Campus, 42130 Konya, Turkey; \\ aktumsek@selcuk.edu.tr \\ 2 Institute of Research and Development, Duy Tan University, Da Nang 550000, Vietnam; \\ mohamadfawzimahomoodally@duytan.edu.vn \\ 3 Department of Health Sciences, Faculty of Science, University of Mauritius, 80837 Réduit, Mauritius; \\ picotcarene@yahoo.com \\ 4 Department for Sustainable Food Process, Università Cattolica del Sacro Cuore, Via Emilia Parmense 84, \\ 29122 Piacenza, Italy; gabriele.rocchetti@unicatt.it (G.R.); luigi.lucini@unicatt.it (L.L.) \\ 5 Chair and Department of Pharmacognosy, Medical University of Lublin, 20-093 Lublin, Poland; \\ esieniawska@pharmacognosy.org \\ 6 Department of Virology, Medical University of Lublin, 20-093 Lublin, Poland; \\ ulubiony.asystent@gmail.com (Ł.Ś.); barbara.rajtar@umlub.pl (B.R.); \\ malgorzata.polz-dacewicz@umlub.pl (M.P.-D.) \\ 7 Department of Pharmaceutical Botany, Pharmacy Faculty, Marmara University, 34854 Istanbul, Turkey; \\ isenkardes@marmara.edu.tr \\ 8 Department of Pharmaceutical Sciences, Food Science and Nutrition Section, University of Perugia, \\ Via S. Costanzo 1, 06126 Perugia, Italy \\ * Correspondence: gokhanzengin@selcuk.edu.tr (G.Z.); domenico.montesano@unipg.it (D.M.)
}

Received: 16 April 2020; Accepted: 27 May 2020; Published: 1 June 2020

\begin{abstract}
Fibigia clypeata (L.) Medik. is a poorly studied plant species belonging to the Brassicaceae family, and usually used as cress in the salads. The current investigation aimed at assessing the antioxidant potential and inhibitory activity of ethyl acetate, methanol, and aqueous extracts of F. clypeata against key enzymes targeted in the management of type II diabetes ( $\alpha$-amylase and $\alpha$-glucosidase), Alzheimer's disease (acetylcholinesterase and butyrylcholinesterase), and skin hyperpigmentation (tyrosinase). Cytotoxicity of the extracts was also determined using normal VERO and cancer FaDu and SCC-25 cell lines. Besides, LC-MS was employed to investigate the detailed phytochemical profiles of the extracts. The methanol extract showed potent enzyme inhibitory activity (4.87 mg galantamine equivalent/g, $3.52 \mathrm{mg}$ galantamine equivalent $/ \mathrm{g}, 126.80 \mathrm{mg}$ kojic acid equivalent/g, and $24.68 \mathrm{mg}$ acarbose equivalent/g, for acetylcholinesterase, butyrylcholinesterase, tyrosinase, and $\alpha$-glucosidase, respectively) and antioxidant potential (96.52, 109.10, 154.02, and $104.85 \mathrm{mg}$ trolox equivalent/g, for DPPH, ABTS, CUPRAC, and FRAP assays, respectively). Interestingly, caffeic acid-O-hexoside derivative, caffeyl alcohol $O$-glucopyranoside, and ferulic acid derivative were identified in all extracts. F. clypeata extracts showed no cytotoxicity towards VERO cell line and a weak cytotoxic potential against FaDu and SCC-25 cell lines. Interesting scientific evidence gathered from the present study support further investigation on $F$. clypeata in the view of designing and developing a novel therapeutic agent for the management of Alzheimer's disease, type II diabetes, skin hyperpigmentation problems, as well as cancer.
\end{abstract}


Keywords: Fibigia clypeata; antioxidants; enzyme inhibition; cytotoxicity; mass spectrometry

\section{Introduction}

Ethnopharmacological literature compiled by our ancestors has indicated the potential of plants in the treatment of several health complications affecting mankind. The therapeutic properties of plants have been ascribed to biologically active chemical compounds called secondary metabolites. Alkaloids, terpenoids, and phenolic compounds are the different classes of secondary metabolites, which naturally occur in plants and have been found to exhibit biological effects, such as anti-inflammatory, anti-cancer, anti-diabetic, and antioxidant properties, amongst others [1-4]. Besides, some of these bioactive compounds (such as polyphenols) possess an important role as dietary constituents, characterized by several health-promoting properties. In the last years, the quest for novel lead candidates for the management of human ailments continues to fuel the study of bioactive compounds from both plants and plant-foods for human nutrition. In particular, several studies have been conducted to test the antioxidant and pharmacological activities of different plant extracts (such as hydroalcoholic, water or ethyl acetate extracts) [5-7].

In this direction, the genus Fibigia, belonging to the Brassicaceae family, is distributed in Egypt, Southern Europe, Caucasus, and the Middle East. Brassicaceae, formerly known as Cruciferae because of their 4-petalled flowers, are a very useful edible group of plants. They encompass many edible plants like broccoli, mustard greens, cauliflower, cabbage, kale, collards, turnips, Chinese Bok Choy, and so on. Many important oil-producing plants are in this family. They also tend to have a pungent taste, as in the mustard greens and seeds, and cress. Besides, one cup of a decoction prepared from the leaves and stem of F. eriocarpa (DC.) Boiss. is used against the common cold [8]. However, to date, few information exists regarding the use of F. clypeata as food ingredient; in fact, to the best of our knowledge, the edible part of this plant (also known as paper pumpkinseed) is the young leaf. In particular, the raw leaves are eaten in the eastern Mediterranean as part of salads. No additional information is provided in the scientific literature regarding other uses as a food ingredient. Overall, a decoction prepared from the stem and fruits of $F$. clypeata (L.) Medik. is used against kidney stones [9], whilst powdered fruits of F. macrocarpa Boiss. are used against cattle infertility [10]. F. clypeata extracts were previously reported exhibiting anti-leishmanial activities on the intracellular amastigote form of the parasite and induced nitrous oxide production by human macrophages [11]. Therefore, according to the literature, the comprehensive chemical characterization, together with the description of other biological properties (such as enzyme inhibitory and/or anti-cancer potential) of the Fibigia species, is still scarce.

Considering the importance of plant bioactive compounds as related to health-promoting attributes, several recent works analyzed the novel source of phytochemicals by using high-resolution targeted/untargeted mass spectrometry approaches [4,6,7]. In fact, according to the literature [12], using liquid chromatography coupled with mass spectrometry (LC-MS) is recommended to profile and then quantify antioxidant compounds (such as polyphenols) in both plant and food matrices. Therefore, the main goal of this work was to assess the potential enzyme inhibitory activity, in vitro antioxidant properties, and cytotoxicity of the ethyl acetate, methanol, and aqueous extract of $F$. clypeata. Moreover, LC-MS was used to elucidate the detailed phytochemical profile of the different extracts. It is expected that the data generated from this study will provide for the first time valuable and comprehensive scientific information on the poorly studied Fibigia species, serving as the baseline information for future detailed investigations. 


\section{Material and Methods}

\subsection{Plant Material and Preparation of Extracts}

The plant material of F. clypeata was collected in the area of Hanönü village (Kastamonu, Turkey) in the summer of 2019. Taxonomic identification was performed by the botanist Dr. Ismail Senkardes (Marmara University, Department of Pharmaceutical Botany, Istanbul, Turkey), and 1 voucher specimen was deposited at the herbarium of Selcuk University (MARE-19856). The grinding of naturally dried aerial parts of the plant was carried out by a laboratory mill.

For the extraction step, the maceration technique based on two different organic solvents, namely ethyl acetate (EA) and methanol. For this purpose, samples of the plant material (5 g) were macerated with $100 \mathrm{~mL}$ of each solvent for $24 \mathrm{~h}$ at room temperature (about $25^{\circ} \mathrm{C}$ ). Then, the solvents were evaporated under vacuum using a rotary evaporator. The aqueous extract was prepared by traditional infusion technique, and plant material $(5 \mathrm{~g})$ was kept with the boiled water $(100 \mathrm{~mL})$ for $20 \mathrm{~min}$. Then the water extract was filtered and then lyophilized. All extracts were stored at $+4{ }^{\circ} \mathrm{C}$ until analysis.

\subsection{Profiling of Bioactive Compounds in the Different Extracts}

To determine total phenolic and flavonoid contents of F. clypeata extracts, colorimetric methods were used based on our previous work [13]. In this regard, the results were finally expressed as namely gallic acid equivalents (GAE) for total phenolics and rutin equivalents (RE) for total flavonoids.

Thereafter, the phytochemical analysis of each plant extract was carried out using Agilent 1200 Infinity HPLC and Agilent 6530B QTOF spectrometer (Agilent Technologies, Santa Clara, CA, USA). The conditions of the analyses were described previously [14]. The identification was based on the obtained fragmentation patterns, which were compared to the data available in the scientific literature and the Metlin database (https://metlin.scripps.edu).

\subsection{Determination of Antioxidant and Enzyme Inhibitory Effects}

To detect antioxidant properties, several chemical assays were used, including different mechanisms, namely, radical scavenging, reducing power, and metal chelating. Trolox (TE) and ethylenediaminetetraacetic acid (EDTA) were used as standard antioxidant compounds. Obtained results were expressed as equivalents of these compounds, Grochowski, et al. [15]. To detect inhibitory effects on enzymes, colorimetric enzyme inhibition assays were used, and these assays included tyrosinase, $\alpha$-glucosidase, $\alpha$-amylase, and cholinesterases. Some standard inhibitors (galantamine, kojic acid, and acarbose) were used as positive controls.

\subsection{Cell Assays}

\subsubsection{Cell Lines and Reagents}

In vitro assays were carried out using normal VERO (ECACC, No. 84113001) and cancer FaDu (ATCC, HTB-43) and SCC-25 (ATCC, CRL-1628) cell lines. Cell media used in experiments, antibiotic supplement (Penicillin-Streptomycin Solution), and PBS (phosphate buffer saline) were provided by Corning (Tewksbury, MA, USA), and Foetal Bovine Serum (FBS) by Biowest (Nuaillé, France). The DMF (dimethylformamide) was acquired from Avantor Performance Materials Poland S.A (Gliwice, Poland). The DMSO (dimethyl sulfoxide), MTT (3-(4,5-dimethylthiazol-2-yl)-2,5-diphenyltetrazolium bromide), and hydrocortisone were purchased from Sigma-Aldrich (St. Louis, MO, USA) and SDS (sodium dodecyl sulfate) from AppliChem (AppliChem GmbH, Darmstadt, Germany). 


\subsubsection{Cell Cultures}

The VERO cells were maintained using DMEM (Dulbecco's Modified Eagle Medium), FaDu cells using Modified Eagle Medium (MEM), whereas SCC-25 required Dulbecco's Modified Eagle Medium/Nutrient Mixture F-12 (DMEM-F12) supplemented with hydrocortisone. Cell propagation was performed using 10\% FBS in appropriate cell media (growth media), whereas the experiments were carried out using media supplemented with $2 \%$ FBS. All cell lines were cultivated in media supplemented with Penicillin-Streptomycin Solution and the propagation of cells was carried out at $37^{\circ} \mathrm{C}$ in $5 \% \mathrm{CO}_{2}$ atmosphere $\left(\mathrm{CO}_{2}\right.$ incubator, Panasonic Healthcare Co., Ltd., Tokyo, Japan).

\subsubsection{Evaluation of Cytotoxic Properties}

The viability of tested cells was assessed using a modified MTT (microculture tetrazolium) test, which measures the ability of succinate dehydrogenase to reduce MTT salt into formazan. Formazan crystals are insoluble in water and are dissolved using a mixture of SDS (14\%), DMF (36\%), and PBS $(50 \%)$, producing a purple color. Usually, after overnight incubation, the absorbance was measured at 2 wavelengths- 540 and $620 \mathrm{~nm}$. The succinate dehydrogenase activity was correlated with the metabolic activity of the cells allowing to assess the viability of the cells [16]. The stock solutions were obtained by dissolving the samples in DMSO $(50 \mathrm{mg} / \mathrm{mL})$ and sterilized using syringe filters $(0.2 \mu \mathrm{m})$. Trypsinized VERO, FaDu, or SCC-25 cells were suspended in appropriate growth media in the density of $1.5 \times 10^{5}$ cells $/ \mathrm{mL}, 2 \times 10^{5}$ cells $/ \mathrm{mL}$ or $3 \times 10^{5}$ cells $/ \mathrm{mL}$, respectively, and cultured in 96-well plates (Corning). After overnight pre-incubation required for obtaining a semi-confluent cell monolayer, the cells were treated for $72 \mathrm{~h}$ with tested extracts in culture media containing $2 \%$ FBS in concentrations varying from 1.95 to $1000 \mu \mathrm{g} / \mathrm{mL}$. Control cells were grown in medium containing $2 \%$ of FBS. The cytotoxicity of DMSO used as a sample solvent was also evaluated. After the incubation, all the media was removed. The plates were rinsed with sterile PBS and $100 \mu \mathrm{L}$ per well of $10 \%$ of MTT solution $(5 \mathrm{mg} / \mathrm{mL})$ in appropriate media was added. After $4 \mathrm{~h}$ of incubation, $100 \mu \mathrm{L}$ per well of solvent containing sodium dodecyl sulfate (14\%), dimethylformamide (36\%), and phosphate buffer saline (50\%) was added. The next day, the absorbance was measured with the use of an Epoch reader (BioTek Instruments, Inc., Winooski, VT, USA) running Gen5 program (2.01.14; BioTek) and data were exported to the GraphPad Prism (v8.0.1) for further analysis. The viability of cells treated with tested samples was compared with control cells, and the values of $\mathrm{CC}_{50}$ (concentration resulting in $50 \%$ inhibition) and $\mathrm{CC}_{10}$ (concentration resulting in 10\% inhibition) were estimated based on dose-response curves.

\subsection{Statistical Analysis}

The analysis of the variance (one-way ANOVA) followed by Duncan's Multiple Range post hoc test $(p<0.05)$ was done using the data from in vitro spectrophotometric and enzymatic assays. In particular, the software PASW Statistics 26.0 (SPSS Inc., Chicago, IL, USA) was used. Therefore, means without a common superscript letter showed significant differences $(p<0.05)$. Pearson's correlations ( $p<0.05$; two-tailed) were then calculated using PASW Statistics 26.0. Regarding the cell assays, the tests were carried out in triplicate and rerun at least thrice. For statistical evaluation, a 2-way ANOVA followed by Tukey's multiple comparison test was used.

\section{Results and Discussion}

\subsection{Phytochemical Profile Determination}

In this work, standard in vitro spectrophotometric analyses was used to determine the total phenolic and flavonoid contents of the ethyl acetate, methanolic, and aqueous extracts of $F$. clypeata. The results are presented in Table 1. 
Table 1. Total bioactive components in the tested extracts. Values are expressed as the mean $(n=3) \pm$ standard deviation. GAE: Gallic acid equivalent; RE: Rutin equivalent. Different superscript letters in the same column indicate significant differences $(p<0.05)$, as determined by Duncan's post-hoc test.

\begin{tabular}{ccc}
\hline Extracts & Total Phenolic Content (mg GAE/g) & Total Flavonoid Content (mg RE/g) \\
\hline Ethyl acetate & $21.04 \pm 0.68^{\mathrm{a}}$ & $10.13 \pm 0.39^{\mathrm{b}}$ \\
Methanolic & $33.42 \pm 3.79^{\mathrm{b}}$ & $21.58 \pm 0.16^{\mathrm{c}}$ \\
Water & $24.43 \pm 0.21^{\mathrm{a}}$ & $5.81 \pm 0.07^{\mathrm{a}}$ \\
\hline
\end{tabular}

As can be observed from the table, the highest phenolic (33.42 mg GAE/g) and flavonoid (21.58 mg RE/g) contents were obtained when considering the methanolic extract. Besides, a strong correlation coefficient $(p<0.01)$ of 0.809 was found between the two different assays (Supplementary Material Table S1). In our previous study on F. eriocarpa [16], the highest total phenolic level was determined in ethyl acetate extract (41.87 mg GAE/g), followed methanol (35.42 mg GAE/g) and water (33.63 mg GAE/g). Similar to current results, methanol extract (24 mg RE/g) of F. eriocarpa contained the highest level of flavonoids. In accordance with our results, several researchers reported that methanol was one of the best solvents to extract flavonoids [17-19]. However, spectrophotometric analyses are mainly able to provide an insight into the content of the bioactive compound of herbal extracts without providing detailed phytochemical composition. Besides, a possible interference of non-phenolic compounds characterizing the extracts has been reported yielding false-positive results [12]. Therefore, in the present study, high performance liquid chromatography coupled to mass spectrometry was used to assess the detailed profile of $F$. clypeata extracts. The identification of compounds was performed by HPLC-ESI-QTOF mass spectrometry in negative ionization mode. The tentatively identified compounds from the extracts were summarized in Table 2, and chromatograms were reported in Figure 1.

Overall, in our experimental conditions, 51 compounds were tentatively identified, mainly consisting of polyphenols and phenolic derivatives, such as flavonoids and phenolic acids. Interestingly, we also found two glucosinolates (i.e., compounds 4 and 5), namely $p$-Methoxy-2-hydroxy-2-phenylethyl glucosinolate and $p$-Methoxy-2-hydroxy-2-phenylethyl glucosinolate-desulfo, previously described in crucifer seeds [22]. In addition, 1, 43, and 57, corresponding to a caffeic acid-O-hexoside derivative, a caffeyl alcohol $O$-glucopyranoside, and a ferulic acid derivative, respectively. These latter were tentatively identified in all the extracts tested. In addition, compounds $\mathbf{6}$ and $\mathbf{7 , 8}$ and $\mathbf{9}$, exhibited the same fragmentation patterns and were characterized from the water extract as dihydroxybenzoic acid hexosides and $p$-coumaric acid ethyl ester derivatives. The fragmentation of compound 16 corresponded to $p$-coumaric acid hexoside, which was tentatively identified from the ethyl acetate extract. Compound 31, with [M-H]- at $m / z$ 193, was identified as ferulic acid. Moreover, several sesquiterpenes (i.e., compounds $\mathbf{4 4}, \mathbf{4 6}$, and $\mathbf{5 1}$ ) were also tentatively characterized (Table 2). 
Table 2. Tentative identification of phytochemical compounds characterizing the different extracts, as resulted by HPLC-ESI-QTOF-MS analysis.

\begin{tabular}{|c|c|c|c|c|c|c|c|c|}
\hline Compound & Tentative Identification & RT (Min) & $\begin{array}{c}\text { Molecular } \\
\text { Formula }\end{array}$ & $\begin{array}{c}\text { Molecular } \\
\text { Weight }\end{array}$ & {$[\mathrm{M}-\mathrm{H}]$} & Fragments $(m / z)$ & Extracts & References \\
\hline 1 & Caffeic acid-O-hexoside derivative & 1.725 & & 388.1247 & 387.1247 & $341.1180 ; 179.0121$ & $\mathrm{EA}, \mathrm{MeOH}$, Water & [20] \\
\hline 2 & Ethyl 2-hydroxy-3-(hydroxyphenyl)propanoate & 2.120 & $\mathrm{C}_{11} \mathrm{H}_{14} \mathrm{O}_{4}$ & 210.0051 & 209.0051 & $\begin{array}{c}\text { 190.9851; } 172.9663 ; 146.9663 \\
\text { 128.9377; } 102.9125\end{array}$ & Water & [21] \\
\hline 3 & Quinic acid & 2.773 & $\mathrm{C}_{7} \mathrm{H}_{12} \mathrm{O}_{6}$ & 192.0205 & 191.0205 & $\begin{array}{l}\text { 173.0034; } 127.0437 ; 111.0463 \\
388.0458 ; 274.9727 ; 259.0033\end{array}$ & $\mathrm{MeOH}$, Water & {$[20]$} \\
\hline 4 & p-Methoxy-2-hydroxy-2-phenylethyl glucosinolate & 4.825 & $\mathrm{C}_{16} \mathrm{H}_{23} \mathrm{NO}_{11} \mathrm{~S}_{2}$ & 469.0042 & 468.0042 & $\begin{array}{c}240.9840 ; 225.9687 ; 194.9916 \\
139.3081 ; 135.9009\end{array}$ & $\mathrm{MeOH}$, Water & [22] \\
\hline 5 & $p$-Methoxy-2-hydroxy-2-phenylethyl glucosinolate-desulfo & 5.018 & $\mathrm{C}_{16} \mathrm{H}_{23} \mathrm{NO}_{8} \mathrm{~S}$ & 389.0527 & 388.0455 & $\begin{array}{c}274.9727 ; 259.0033 ; 240.9840 ; \\
225.9687 ; 194.9916 ; \\
139.3081 ; 135.9009\end{array}$ & $\mathrm{MeOH}$, Water & [22] \\
\hline 6 & Dihydroxybenzoic acid hexoside & 15.386 & $\mathrm{C}_{13} \mathrm{H}_{16} \mathrm{O}_{9}$ & 316.0741 & 315.0741 & $153.0440 ; 109.0311$ & Water & [20] \\
\hline 7 & Dihydroxybenzoic acid hexoside & 17.781 & $\mathrm{C}_{13} \mathrm{H}_{16} \mathrm{O}_{9}$ & 316.0772 & 315.0772 & $153.0440 ; 109.0311$ & Water & [20] \\
\hline 8 & $p$-Coumaric acid ethyl ester derivative & 18.460 & & 314.0613 & 313.0613 & $\begin{array}{r}191.0031 ; 172.9730 \\
1146.9803 ; 128.9525\end{array}$ & Water & [20] \\
\hline 9 & $p$-Coumaric acid ethyl ester derivative & 19.622 & & 314.0613 & 313.0613 & $\begin{array}{l}191.9672 ; 172.9696 \\
146.9676 ; 128.9426\end{array}$ & Water & {$[20]$} \\
\hline 10 & 2-hydroxy-3-phenylpropanoic acid hexoside & 20.062 & $\mathrm{C}_{15} \mathrm{H}_{20} \mathrm{O}_{8}$ & 328.1154 & 327.1154 & $\begin{array}{l}165.0021 ; 146.9606 ; \\
128.9594 ; 100.9476\end{array}$ & EA & [23] \\
\hline 11 & $\begin{array}{l}\text { Rhamnoside of ethyl } \\
\text { 2-hydroxy-3-(hydroxyphenyl)propanoate isomer }\end{array}$ & 20.466 & $\mathrm{C}_{17} \mathrm{H}_{24} \mathrm{O}_{8}$ & 356.0780 & 355.0780 & $\begin{array}{l}\text { 209.0037; 191.0097; } 181.3211 \\
\text { 163.0022; } 146.9911\end{array}$ & Water & [23] \\
\hline 12 & $\begin{array}{l}\text { Rhamnoside of ethyl } \\
\text { 2-hydroxy-3-(hydroxyphenyl)propanoate isomer }\end{array}$ & 21.598 & $\mathrm{C}_{17} \mathrm{H}_{24} \mathrm{O}_{8}$ & 356.0780 & 355.0780 & $\begin{array}{l}209.0401 ; 191.0162 ; 181.3317 \\
163.0043 ; 146.9924\end{array}$ & Water & [23] \\
\hline 13 & Benzoic acid & 21.641 & $\mathrm{C}_{7} \mathrm{H}_{6} \mathrm{O}_{2}$ & 122.0369 & 121.0369 & - & EA & [23] \\
\hline 14 & Feruloyl, ethyl 2-hydroxy-3-(hydroxyphenyl)propanoate & 22.395 & $\mathrm{C}_{17} \mathrm{H}_{22} \mathrm{O}_{10}$ & 386.0912 & 385.0912 & $\begin{array}{l}209.0082 ; 190.9877 ; \\
146.9696 ; 129.9443\end{array}$ & Water & [23] \\
\hline 15 & Sinapic acid hexoside derivative & 22.488 & & 432.2047 & 431.2047 & $385.2424 ; 223.1204$ & $\mathrm{MeOH}$, Water & {$[24,25]$} \\
\hline 16 & $p$-Coumaric acid hexoside & 22.687 & $\mathrm{C}_{15} \mathrm{H}_{18} \mathrm{O}_{8}$ & 326.0987 & 325.0987 & 162.9889.;144.9626; 119.0003 & EA & {$[20]$} \\
\hline 17 & $p$-Coumaric acid ethyl ester derivative & 23.203 & & 314.0613 & 313.0613 & $\begin{array}{l}\text { 190.9426; } 172.9625 ; 146.9600 ; \\
\text { 128.9344; } 120.9409\end{array}$ & Water & [20] \\
\hline 18 & $p$-coumaroyl acetic acid derivative & 23.407 & & 388.1763 & 387.1763 & 207.0850; 163.0775 & $\mathrm{MeOH}$ & [21] \\
\hline 19 & Protocatechuic acid derivative & 23.613 & & 432.2013 & 431.2013 & $385.1909 ; 223.1168 ; 153.0360$ & EA & [21] \\
\hline 20 & $\begin{array}{l}\text { Rhamnoside of ethyl } \\
\text { 2-hydroxy-3-(hydroxyphenyl)propanoate }\end{array}$ & 23.976 & $\mathrm{C}_{17} \mathrm{H}_{24} \mathrm{O}_{8}$ & 356.0780 & 355.0780 & $209.0074 ; 190.9903$ & Water & [23] \\
\hline 21 & (Epi)catechin & 24.569 & $\mathrm{C}_{15} \mathrm{H}_{14} \mathrm{O}_{6}$ & 290.0440 & 289.0440 & $245.0665 ; 205.0202 ; 125.9405$ & $\mathrm{MeOH}$ & {$[20]$} \\
\hline 22 & Phenylacetic acid & 24.773 & $\mathrm{C}_{8} \mathrm{H}_{8} \mathrm{O}_{2}$ & 136.0057 & 135.0057 & - & EA & [20] \\
\hline 23 & Feruloyl, ethyl 2-hydroxy-3-(hydroxyphenyl)propanoate & 24.994 & $\mathrm{C}_{17} \mathrm{H}_{22} \mathrm{O}_{10}$ & 386.0912 & 385.0912 & $\begin{array}{l}\text { 223.01146; 209.0082; } 190.9877 ; \\
\text { 146.9696; } 129.9443\end{array}$ & Water & [23] \\
\hline 24 & O-sinapoyl-glucose & 25.870 & $\mathrm{C}_{17} \mathrm{H}_{22} \mathrm{O}_{10}$ & 386.1268 & 385.1268 & 223.0670; & $\mathrm{MeOH}$, Water & [25] \\
\hline 25 & Vanilic acid derivative hexoside & 26.244 & & 508.2029 & 507.2029 & $\begin{array}{l}345.1089 ; 327.1215 ; \\
315.1409 ; 167.0116\end{array}$ & EA & [21] \\
\hline 26 & Ellagic acid & 26.397 & $\mathrm{C}_{14} \mathrm{H}_{6} \mathrm{O}_{8}$ & 302.1221 & 301.1221 & $\begin{array}{l}283.1089 ; 271.1000 ; \\
160.9988 ; 125.9795\end{array}$ & EA & {$[21]$} \\
\hline 27 & Sinapic acid derivative & 27.423 & & 312.1533 & 311.1533 & $223.8222 ; 208.0325$ & EA & {$[24,25]$} \\
\hline 28 & Caffeic acid derivative & 28.138 & & 416.2092 & 415.2092 & $179.0079 ; 137.0253$ & $\mathrm{MeOH}$ & [21] \\
\hline
\end{tabular}


Table 2. Cont.

\begin{tabular}{|c|c|c|c|c|c|c|c|c|}
\hline Compound & Tentative Identification & RT (Min) & $\begin{array}{c}\text { Molecular } \\
\text { Formula }\end{array}$ & $\begin{array}{l}\text { Molecular } \\
\text { Weight }\end{array}$ & {$[\mathrm{M}-\mathrm{H}]$} & Fragments $(m / z)$ & Extracts & References \\
\hline 29 & Quercetin-O-hexoside & 29.159 & $\mathrm{C}_{21} \mathrm{H}_{20} \mathrm{O}_{12}$ & 464.1014 & 463.1014 & $301.0300 ; 178.9275 ; 150.9379$ & $\mathrm{MeOH}$ & [20] \\
\hline 30 & Dimethoxy-trihydroxyflavone & 30.177 & $\mathrm{C}_{17} \mathrm{H}_{14} \mathrm{O}_{7}$ & 300.1051 & 299.1051 & 269.0865 & EA & [23] \\
\hline 31 & Ferulic acid & 30.672 & $\mathrm{C}_{10} \mathrm{H}_{10} \mathrm{O}_{4}$ & 194.0153 & 193.0153 & $160.9938 ; 151.0017 ; 133.9934$ & $\mathrm{MeOH}$ & {$[21,23]$} \\
\hline 32 & Kaempferol glucoside & 30.710 & $\mathrm{C}_{21} \mathrm{H}_{20} \mathrm{O}_{11}$ & 448.1060 & 447.1060 & 285.0817 & $\mathrm{MeOH}$ & [14] \\
\hline 33 & Caffeic acid derivative & 30.788 & & 362.1790 & 361.1790 & $\begin{array}{l}\text { 193.0563; } 179.0297 ; 165.0047 \\
146.9725 ; 135.9938 ; 120.9468\end{array}$ & Water & [20] \\
\hline 34 & Quercetin derivative & 30.905 & & 550.0995 & 549.0995 & $505.1463 ; 301.0477$ & $\mathrm{MeOH}$ & [20] \\
\hline 35 & Gallic acid derivative & 31.085 & & 188.0603 & 187.0603 & $169.0445 ; 125.0163$ & EA & [21] \\
\hline 36 & Caffeic acid derivative & 32.040 & & 362.1765 & 361.1765 & $\begin{array}{l}301.1126 ; 285.2249 ; 179.0294 \\
165.0075 ; 146.9873: 120.9487\end{array}$ & EA & [21] \\
\hline 37 & Unidentified & 32.203 & & 306.0734 & 305.0734 & $\begin{array}{c}165.0075 ; 146.9875 ; 120.9487 \\
225.0952\end{array}$ & $\mathrm{MeOH}$, Water & \\
\hline 38 & Unidentified dimer & 33.506 & & 896.3362 & 895.3362 & $447.1766 ; 403.1865 ; 343.1615$ & $\mathrm{MeOH}$, Water & \\
\hline 39 & Dihydrocoumaric acid derivative & 34.565 & & 246.0071 & 244.9989 & 165.0111 & $\mathrm{MeOH}$, Water & [20] \\
\hline 40 & Trihydroxy-methoxyflavone derivative hexoside & 35.032 & & 920.2537 & 919.2537 & $\begin{array}{l}\text { 757.2071; } 461.1288 ; \\
299.0536 ; 284.0217\end{array}$ & Water & [14] \\
\hline 41 & Unidentified & 36.106 & & 534.1841 & 533.1841 & $\begin{array}{l}445.2067 ; 385.1792 ; \\
343.1590 ; 163.0220\end{array}$ & Water & \\
\hline 42 & Tetrahydroxy-dimethoxyflavone & 36.744 & $\mathrm{C}_{17} \mathrm{H}_{14} \mathrm{O}_{8}$ & 346.0740 & 345.0740 & $330.0496 ; 315.0293$ & Water & [14] \\
\hline 43 & Caffeyl alcohol O-glucopyranoside & 37.102 & $\mathrm{C}_{15} \mathrm{H}_{20} \mathrm{O}_{8}$ & 328.2227 & 327.2227 & $229.1231 ; 165.0727 ; 121.0198$ & $\mathrm{EA}, \mathrm{MeOH}$, Water & [23] \\
\hline 44 & Sesquiterpene-derivative & 38.691 & & 330.2386 & 329.2386 & $\begin{array}{l}\text { 289.1532; 229.1252; } \\
\text { 211.1104: } 129.0162\end{array}$ & $\mathrm{MeOH}$, Water & \\
\hline 45 & Trihydroxy-methoxyflavone & 39.570 & $\mathrm{C}_{16} \mathrm{H}_{12} \mathrm{O}_{6}$ & 300.0572 & 299.0572 & 284.0313 & EA & [14] \\
\hline 46 & Sesquiterpene-derivative & 43.427 & & 308.1944 & 307.1944 & $\begin{array}{r}289.1829 ; 235.1171 ; 209.0913 ; \\
1850827.120 .9816\end{array}$ & $\mathrm{MeOH}$, Water & \\
\hline 47 & Ferulic acid derivative & 44.078 & & 294.1770 & 293.1770 & $236.0898 ; 221.1337 ; 193.1262$ & $\mathrm{EA}, \mathrm{MeOH}$, Water & [21] \\
\hline 48 & Phenylacetic acid derivative & 45.795 & & 306.1786 & 305.1786 & 249.1359; 135.0091; 125.0171; & EA & [20] \\
\hline 49 & Sinapic acid derivative & 45.927 & & 312.2265 & 311.2265 & $223.1479 ; 208.4343$ & $\mathrm{MeOH}$, Water & {$[24,25]$} \\
\hline 50 & Sinapine & 46.770 & $\mathrm{C}_{16} \mathrm{H}_{24} \mathrm{NO}_{5}$ & 310.2099 & 309.2099 & $\begin{array}{l}\text { 291.2122; 251.1664; } \\
\text { 223.1588; 208.6589 }\end{array}$ & EA & [25] \\
\hline 51 & Sesquiterpene-derivative & 47.632 & & 310.2099 & 309.2099 & $\begin{array}{l}\text { 291.1943; 225.1275; } \\
\text { 197.0855; } 110.9822\end{array}$ & EA & \\
\hline
\end{tabular}




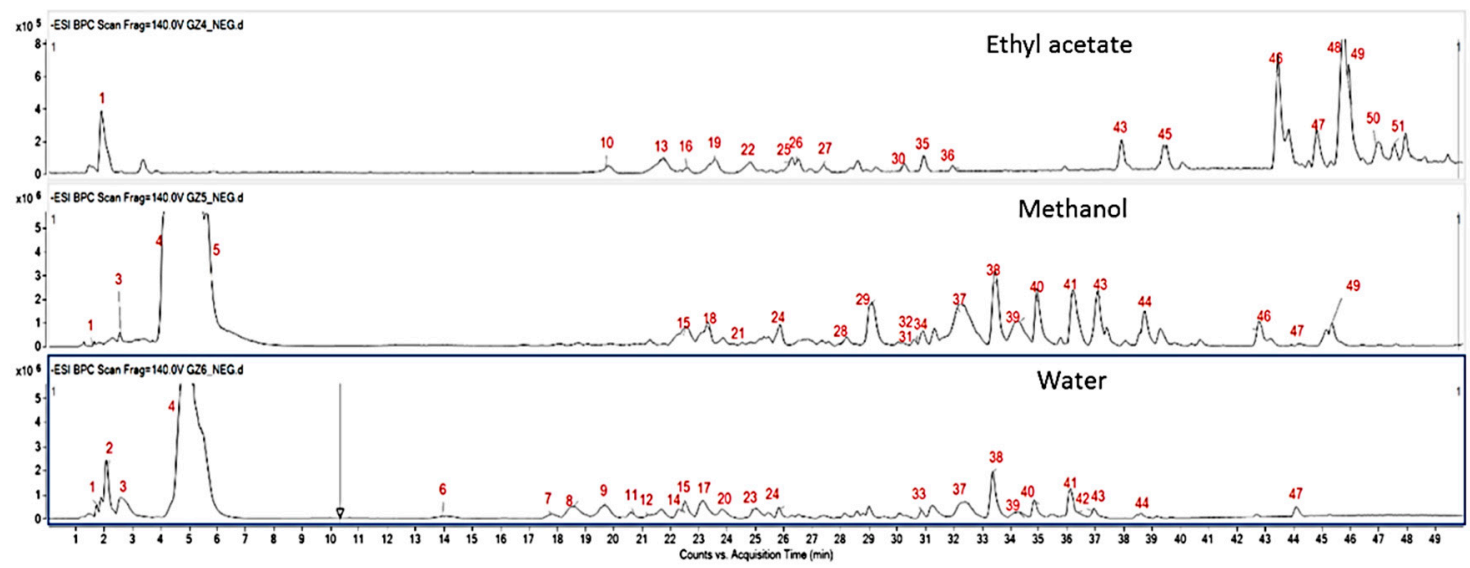

Figure 1. Chromatograms of the different F. clypeata extracts (i.e., ethyl acetate, methanol, and water), as result by HPLC-ESI-QTOF analysis. The numbers of compounds (red color) correspond to those reported in Table 2.

\subsection{Enzyme Inhibition Activity}

Enzyme inhibitors hold a significant share in clinically approved drugs, and their attractiveness has been associated with their specific roles in several metabolic pathways. Drug discovery and development focus on identifying and optimizing lead compounds that act on specific enzymes [26]. In the present study, the ability of F. clypeata extracts to inhibit key enzymes targeted in the management of Alzheimer's disease, skin hyperpigmentation, and type II diabetes was assessed. About 50 million people worldwide have dementia and Alzheimer's disease, the most common form of dementia accounts for $60-70 \%$ of the cases [27]. Based on the cholinergic hypothesis, the lack of neurotransmitters hinders connections between neurons and thus affects the brain's neuronal circuits [28]. The inhibition of acetylcholinesterase has been advocated in the management of mild cognitive impairment due to Alzheimer's disease. Clinically approved drugs, such as donepezil, galantamine, and rivastigmine, containing acetylcholinesterase inhibitor as an active ingredient, are currently used in the management of Alzheimer's disease [29]. Later, the function of another cholinesterase enzyme, namely, butyrylcholinesterase, has also been evoked. The increased activity of butyrylcholinesterase, up to $120 \%$, in the late stage of Alzheimer's disease has been related to the wasting away of the brain and aggravation of behavioral and cognitive dysfunction in Alzheimer's disease patients [30]. Besides, it has been reported that butyrylcholinesterase could compensate a lack of acetylcholinesterase in the acetylcholinesterase knockout mice model [31]. These facts have brought the role and need for butyrylcholinesterase inhibition into the limelight. Finding a novel candidate showing both acetylcholinesterase and butyrylcholinesterase inhibitory activity represents an interesting therapeutic strategy for the management of Alzheimer's disease. However, as reported in the literature, further ad-hoc studies are strongly required to find also possible markers of the neurodegenerative disease [32]. As can be observed from Table 3, the ethyl acetate and methanol extracts of $F$. clypeata showed inhibitory action against both cholinesterase enzymes.

The methanolic extract (4.87 mg GALAE/g) was most active against acetylcholinesterase. Both the ethyl acetate (3.54 mg GALAE/g) and methanol (3.52 mg GALAE/g) extracts showed comparable inhibition against butyrylcholinesterase. In our previous study, the best cholinesterase inhibition abilities of F. eriocarpa were reported for ethyl acetate (2.12 mg GALAE/g for AChE and $2.01 \mathrm{mg}$ GAELAE/g for BChE) and methanol (1.83 mg GALAE/g for AChE and $1.08 \mathrm{mg}$ GALAE/g for BChE) extracts [16]. Based on these values, the ethyl acetate and methanol extracts of $F$. clypeata were stronger than F. eriocarpa. In the literature survey, several researchers reported the significant cholinesterase inhibitory abilities for other solvents (ethyl acetate, chloroform, and methanol, etc.) rather than water [33-35]. This fact could be explained to extract low polarity compounds such as alkaloids and terpenoids with these solvents, and they could be more active on cholinesterases. 
Table 3. Enzyme inhibitory activities of the tested extracts. Values are expressed as mean $(n=3) \pm$ standard deviation. GALAE: Galantamine equivalent; KAE: Kojic acid equivalent; ACAE: Acarbose equivalent; na $=$ not active. Different superscript letters in the same column indicate significant differences ( $p$ textless 0.05), as determined by Duncan's post-hoc test.

\begin{tabular}{|c|c|c|c|c|c|}
\hline Extracts & $\begin{array}{l}\text { AChE Inhibition } \\
\text { (mg GALAE/g) }\end{array}$ & $\begin{array}{l}\text { BChE Inhibition } \\
\text { (mg GALAE/g) }\end{array}$ & $\begin{array}{l}\text { Tyrosinase } \\
\text { Inhibition } \\
\text { (mg KAE/g) }\end{array}$ & $\begin{array}{c}\text { Amylase } \\
\text { Inhibition } \\
\text { (mmol ACAE/g) }\end{array}$ & $\begin{array}{l}\text { Glucosidase } \\
\text { Inhibition } \\
\text { (mmol ACAE/g) }\end{array}$ \\
\hline Ethyl acetate & $3.49 \pm 0.16^{b}$ & $3.54 \pm 0.43^{b}$ & $115.07 \pm 3.14^{b}$ & $0.73 \pm 0.04^{c}$ & $22.32 \pm 1.15^{b}$ \\
\hline Methanolic & $4.87 \pm 0.57^{c}$ & $3.52 \pm 0.11^{b}$ & $126.80 \pm 0.39^{c}$ & $0.55 \pm 0.03^{b}$ & $24.68 \pm 0.03^{c}$ \\
\hline Water & $\mathrm{na}^{\mathrm{a}}$ & $\mathrm{na}^{\mathrm{a}}$ & $43.11 \pm 5.14^{\mathrm{a}}$ & $0.11 \pm 0.01^{\mathrm{a}}$ & $1.06 \pm 0.20^{a}$ \\
\hline
\end{tabular}

Likewise, the methanolic and ethyl acetate extracts of $F$. clypeata showed potent inhibition against tyrosinase. The inhibition of tyrosinase is crucial in the management of skin hyperpigmentation conditions, such as melasma and freckles. Inhibiting tyrosinase is directly related to the reduced production of the dark brown melanin. Moreover, the increased public interest towards naturally derived products, including cosmetic and dermatological products, has fueled the need for natural tyrosinase inhibitors. Another species of the Fibigia genus, namely F. eriocarpa, was previously reported to inhibit tyrosinase [16]. In contrast to our present findings, the ethyl acetate and methanol extracts of F. eriocarpa were not active on tyrosinase. This fact could be explained with the differences of chemical profiles in these extracts and the complex interactions of phytochemicals. However, methanolic extracts exhibited stronger actions on tyrosinase in earlier studies conducted by some researchers [36,37].

Monitoring hyperglycemia, the hallmark of type II diabetes, is playing a pivotal role in the management of the disease. Apart from dietary modifications, the inhibition of carbohydrate hydrolyzing enzymes assists in maintaining a normal glycemic level. The inhibition of $\alpha$-amylase, which is situated in the upper gastrointestinal tract and catalyzes the initial breakdown of ingested polysaccharides into oligosaccharides and the inhibition of $\alpha$-glucosidase, which is situated in the brush border of the small intestine and catalyzes the last step of carbohydrate digestion, significantly prevents glycemic peaks. However, prominent $\alpha$-amylase inhibition has been associated with gastrointestinal discomforts caused by the fermentation of undigested carbohydrates in the colon. In the present study, a low $\alpha$-amylase inhibition was observed while the ethyl acetate $(22.32 \mathrm{mmol} \mathrm{ACAE} / \mathrm{g})$ and methanol (24.68 mmol ACAE/g) extracts of F. clypeata exhibited potent $\alpha$-glucosidase inhibitory action. It was noted that, in general, the water extract of $F$. clypeata exhibited the lowest enzymatic inhibition. The higher activity of the ethyl acetate and methanol extracts might be related to the synergistic action of the different bioactive compounds present in these extracts. Anti-diabetic properties of the ethyl acetate and methanol extracts of $F$. clypeata were stronger than those of F. eriocarpa (amylase: $0.44 \mathrm{mmol}$ ACAE/g for ethyl acetate and $0.43 \mathrm{mmol} \mathrm{ACAE} / \mathrm{g}$ for methanol; glucosidase: $5.01 \mathrm{mmol} \mathrm{ACAE} / \mathrm{g}$ for ethyl acetate and $1.57 \mathrm{mmol} \mathrm{ACAE} / \mathrm{g}$ for methanol) in our previous paper. Additionally, it is worth mentioning the level of the different bioactive compounds might have also affected enzyme activity. The alkaloidal amine, sinapine, tentatively characterized from the ethyl acetate extract of F. clypeata was previously reported to inhibit acetylcholinesterase isolated from rat cerebral homogenate with an IC50 of $3.66 \mu \mathrm{mol} / \mathrm{L}$ [38]. Regarding correlations between total phenolic-flavonoid content with enzymatic assays, we found strong correlation coefficients when considering only TFC (total flavonoid content) values. In this regard, total flavonoids were strongly correlated to $\mathrm{AChE}(0.870 ; p<0.01)$, tyrosinase (0.795; $p<0.05), \alpha$-glucosidase $(0.771 ; p<0.05)$, and BChE $(0.705 ; p<0.05)$ inhibition values (Table S1).

\subsection{In Vitro Antioxidant Activity of the Tested Extracts}

The role of oxidative stress in the onset and/or progression of human ailments supports the systematic antioxidant evaluation of studied plant extracts. Antioxidants can act by different mechanisms, namely, hydrogen atom transfer, single electron transfer, or transition metal chelation [39]. In this study, multiple antioxidant assays were used to obtain a comprehensive understanding of the antioxidant properties of the F. clypeata extracts. As presented in Table 4, the methanolic extract 
(96.52 and $109.10 \mathrm{mg}$ TE/g, for DPPH and ABTS, respectively) of F. clypeata showed potent radical scavenging properties.

Table 4. In vitro antioxidant properties of the tested extracts. Values are expressed as the mean $(n=3)$ \pm standard deviation. TE: Trolox equivalents; EDTAE: EDTA equivalents. Different superscript letters in the same column indicate significant differences $(p<0.05)$, as determined by Duncan's post-hoc test.

\begin{tabular}{ccccccc}
\hline Extracts & $\begin{array}{c}\text { DPPH } \\
(\mathbf{m g ~ T E} / \mathbf{g})\end{array}$ & $\begin{array}{c}\text { ABTS } \\
(\mathbf{m g} \text { TE/g) }\end{array}$ & $\begin{array}{c}\text { CUPRAC } \\
\mathbf{( m g ~ T E} / g)\end{array}$ & $\begin{array}{c}\text { FRAP } \\
\text { (mg TE/g) }\end{array}$ & $\begin{array}{c}\text { Phosphomolybdenum } \\
\text { (mmol TE/g) }\end{array}$ & $\begin{array}{c}\text { Chelating } \\
\text { Ability } \\
(\mathbf{m g} \text { EDTAE/g) }\end{array}$ \\
\hline $\begin{array}{c}\text { Ethyl } \\
\text { acetate }\end{array}$ & $22.38 \pm 0.37^{\mathrm{a}}$ & $15.61 \pm 0.58^{\mathrm{a}}$ & $78.41 \pm 4.04^{\mathrm{a}}$ & $28.53 \pm 1.47^{\mathrm{a}}$ & $1.91 \pm 0.05^{\mathrm{b}}$ & $25.21 \pm 1.20^{\mathrm{b}}$ \\
$\begin{array}{c}\text { Methanolic } \\
\text { Water }\end{array}$ & $96.52 \pm 0.10^{\mathrm{c}}$ & $109.10 \pm 2.57^{\mathrm{c}}$ & $154.02 \pm 4.58^{\mathrm{c}}$ & $104.85 \pm 0.57^{\mathrm{c}}$ & $1.79 \pm 0.15^{\mathrm{b}}$ & $21.18 \pm 1.72^{\mathrm{a}}$ \\
\hline
\end{tabular}

Likewise, the methanolic extract exhibited the highest reducing potent in the CUPRAC (154.02 mg TE/g) and FRAP (104.85 mg TE/g) assays. Obtained results could be compared with earlier studies on other Fibigia or Brassicaceae species. For example, the antioxidant properties in F. eriocarpa extracts can be ranked methanol $>$ ethyl acetate $>$ water. However, the antioxidant abilities of the water extract of $F$. clypeata were stronger than that of F. eriocarpa. [16] Besides, different levels for antioxidant properties in some Brassicaceae species have been reported in the literature [40-42]. At this point, the chemical profiles of the extracts were closely related to their antioxidant properties. In this sense, several compounds present in F. clypeata methanolic extract might be responsible for the observed activity. Sinapic acid was reported to exhibit radical scavenging potential [43]. Caffeic acid has been reported to be an effective radical scavenger and reducing agent as well as a metal chelator [44]. In terms of metal chelating, the aqueous extract of $F$. clypeata showed the highest activity. These findings suggest that bioactive compounds present in the methanolic extract exhibited mostly radical scavenging and reducing potential, while compounds present in the aqueous extract were potential metal chelators. Regarding the potential correlation between TPC and TFC values, we found strong correlation coefficients between TPC and some antioxidant assays, such as DPPH (0.943; $p<0.01)$, CUPRAC (0.954; $p<0.01)$, FRAP (0.874; $p<0.01)$, and ABTS $(0.790 ; p<0.05)$ values. In addition, total flavonoids were inversely correlated to chelating activity $(-0.902 ; p<0.01)$ and strongly correlated to DPPH values $(0.876 ; p<0.01)$ (Table S1).

\subsection{Cell Assays}

To assess the anti-cancer potential of Fibigia clypeata extracts, their cytotoxicity was measured on two cancer cell lines belonging to the group of head and neck squamous cell carcinomas (HNSCC), i.e., squamous cell carcinoma of the pharynx $(\mathrm{FaDu})$ and squamous cell carcinoma of the tongue (SCC-25) and compared with the results obtained for the normal VERO cell line. The results of cytotoxicity evaluation are presented in Table 5.

Table 5. Cytotoxicity potential of the tested extracts. na = not applicable.

\begin{tabular}{cccc}
\hline & \multicolumn{3}{c}{$\mathrm{CC}_{50}(\mu \mathrm{g} / \mathrm{mL})$} \\
\cline { 2 - 4 } & Ethyl Acetate & Methanol & Water \\
\hline VERO & $>500$ & na & na \\
FaDu & $231.6 \pm 13.97$ & $363.13 \pm 19.56$ & $>500$ \\
SCC-25 & $554.57 \pm 62.34$ & $408.43 \pm 48.52$ & $412.43 \pm 51.61$ \\
\hline
\end{tabular}

In the case of VERO cells, no cytotoxicity was observed for methanolic and aqueous extracts. The ethyl acetate extract showed a cytotoxic effect in concentrations above $125 \mu \mathrm{g} / \mathrm{mL}$, but it was impossible to calculate the CC50 value because even in the highest tested concentration of $1000 \mu \mathrm{g} / \mathrm{mL}$ the viability of VERO cells was above $50 \%$ (Figure 2). 
Fibigia clypeata EtOAc extract

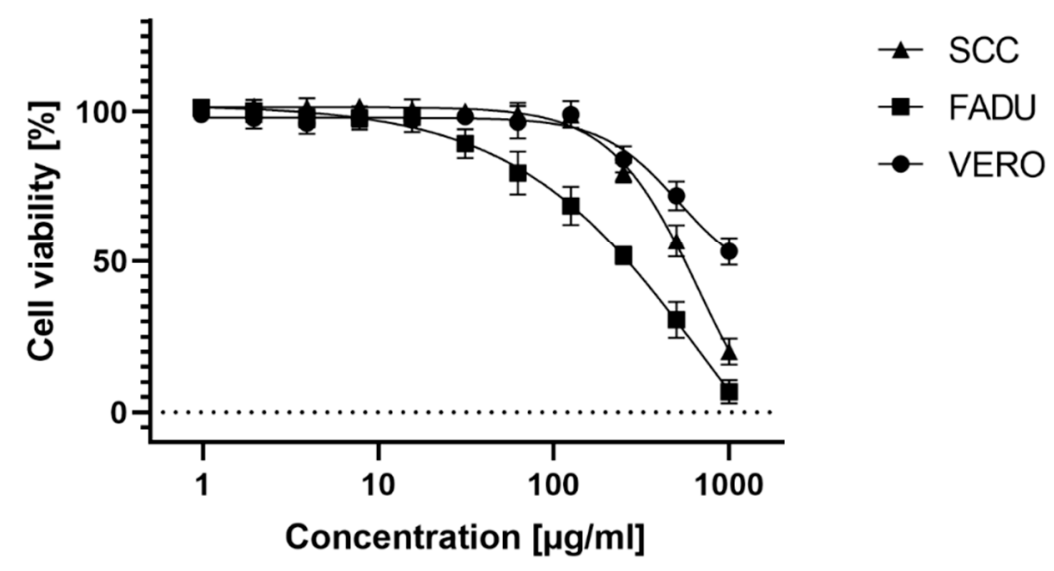

Figure 2. Cytotoxicity of Fibigia clypeata ethyl acetate extract.

Similarly, it was impossible to calculate CC50 for aqueous extract on $\mathrm{FaDu}$ cells. Both ethyl acetate and methanolic extracts showed selective toxicity towards FaDu cells. In the case of SCC- 25 cell line, selective toxicity was observed for all tested extracts. The CC50 and CC10 values of ethyl acetate extract on both cancer cell lines showed a statistically important difference $(p<0.001)$. In the case of methanolic extract tested on FaDu and SCC-25, the CC50 values were similar, but a significant difference was found for the CC10 values $(p<0.001)$ (Figures 3 and 4 ).

Fibigia clypeata MeOH extract

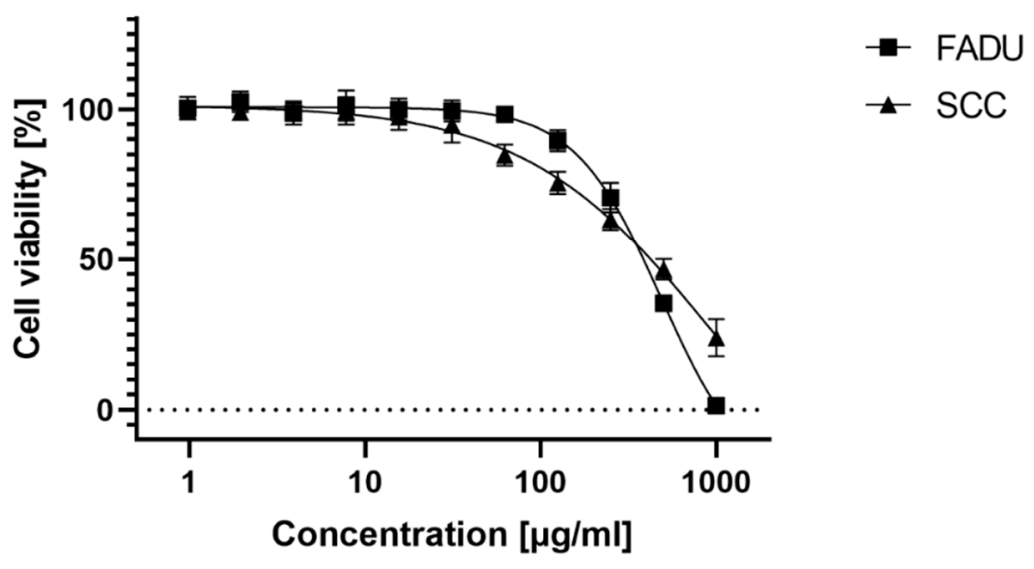

Figure 3. Cytotoxicity of Fibigia clypeata methanolic extract.

The criteria of classification of plant extract cytotoxicity according to the protocols suggested by the National Cancer Institute (NCI) [45] and selected literature are shown in Table 6 [45-48].

Table 6. Classification of plant extracts cytotoxicity according to National Cancer Institute.

\begin{tabular}{ccccc}
\hline $\mathrm{CC}_{50}$ Value & $<20 \mu \mathrm{g} / \mathrm{mL}$ & $21-200 \mu \mathrm{g} / \mathrm{mL}$ & $201-500 \mu \mathrm{g} / \mathrm{mL}$ & $>500 \mu \mathrm{g} / \mathrm{mL}$ \\
\hline Cytotoxic activity & high & moderate & weak & no activity \\
\hline
\end{tabular}

According to this criterion, all tested extracts can be classified as non-cytotoxic towards the VERO cell line. In the case of cancer cell lines, ethyl acetate and methanolic extracts tested on FaDu and methanolic and aqueous extracts tested on SCC- 25 can be classified as weakly cytotoxic. However, the selective activity towards cancer cell lines encourages further investigations focused on the isolation 
of bioactive constituents and testing their anti-cancer potential. Di Giorgio et al. [11] studied the immunomodulatory and anti-leishmanial activities of Lebanese plants and reported the cytotoxicity of F. clypeata extracts assessed on the THP1 human monocyte cell line by colorimetric determination of cell viability using the oxidation-reduction indicator Alamar Blue. The aqueous and dichloromethane extracts showed CC50 of 124.9 and $123.6 \mu \mathrm{g} / \mathrm{mL}$, whereas in the case of methanol extract, it was above $250 \mu \mathrm{g} / \mathrm{mL}$.

\section{$\mathrm{CC}_{10}[\mu \mathrm{g} / \mathrm{mL}]$}

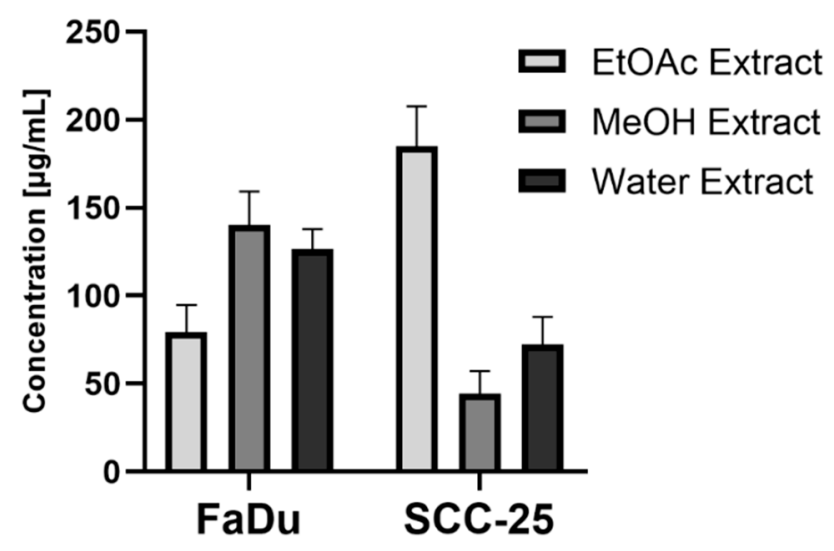

Figure 4. The CC10 values of Fibigia clypeata extracts on cancer cell lines.

\section{Conclusions}

The present work presents interesting scientific data on the potential of Fibigia clypeata in the management of Alzheimer's disease, type II diabetes, and skin hyperpigmentation problems. Cytotoxicity studies also supported the action of $F$. clypeata against cancerous cell lines, thereby advocating further investigation geared towards assessing the anti-cancer properties of F. clypeata. Higher enzyme inhibition and in vitro antioxidant capabilities were noted for the methanolic extract, showing that the choice of solvent greatly affected the extraction of bioactive compounds from the plant material. Therefore, considering the potential health-implication given by this plant-food for human nutrition, both in vivo and bioavailability studies appear to be strongly required to further support the preliminary data collected in the present study.

Supplementary Materials: The following are available online at http://www.mdpi.com/2304-8158/9/6/705/s1, Table S1: Pearson's correlation coefficients considering the different in vitro spectrophotometric and enzymatic assays.

Author Contributions: Conceptualization, G.Z., M.F.M., E.S., methodology, G.Z., G.R., E.S., Ł.Ś. B.R. M.P.-D., software, G.Z. and G.R., validation, G.Z., G.R., L.L., formal analysis: G.Z, G.R., I.S., A.A., investigation, G.Z., M.F.M., G.R., A.A., M.C.N.P.-A., resources, G.Z., A.A., D.M., data curation, G.Z., M.F.M., G.R., L.L., Ł.Ś., B.R., M.P.-D., I.S., M.C.N.P.-A., writing—original draft preparation, G.Z., M.F.M., G.R., Ł.Ś., B.R., M.P.-D., I.S. writing一review and editing, G.Z., M.F.M., G.R., L.L., D.M., visualization, G.Z., supervision, L.L. and D.M. project administration, G.Z., and A.A., funding acquisition, A.A., D.M. All authors have read and agreed to the published version of the manuscript.

Funding: This research received no external funding.

Conflicts of Interest: The authors declare no conflict of interest.

\section{References}

1. Borbone, N.; Borrelli, F.; Montesano, D.; Izzo, A.A.; De Marino, S.; Capasso, R.; Zollo, F. Identification of a new sesquiterpene polyol ester from Celastrus paniculatus. Planta Med. 2007, 73, 792-794. [CrossRef]

2. De Almeida, E.M.; Ferreira, H.J.; Alves, D.R.; da Silva, W.M.B. Therapeutic potential of medicinal plants indicated by the Brazilian public health system in treating the collateral effects induced by chemotherapy, radiotherapy, and chemoradiotherapy: A systematic review. Complement. Ther. Med. 2020, 49, 102293. [CrossRef] 
3. Rocchetti, G.; Giuberti, G.; Busconi, M.; Marocco, A.; Trevisan, M.; Lucini, L. Pigmented sorghum polyphenols as potential inhibitors of starch digestibility: An in vitro study combining starch digestion and untargeted metabolomics. Food Chem. 2020, 312, 126077. [CrossRef]

4. Senizza, B.; Rocchetti, G.; Okur, M.A.; Zengin, G.; Yıldıztugay, E.; Ak, G.; Montesano, D.; Lucini, L. Phytochemical Profile and Biological Properties of Colchicum triphyllum (Meadow Saffron). Foods 2020, 9, 457. [CrossRef]

5. Borrelli, F.; Borbone, N.; Capasso, R.; Montesano, D.; De Marino, S.; Aviello, G.; Aprea, G.; Masone, S.; Izzo, A.A. Potent relaxant effect of a Celastrus paniculatus extract in the rat and human ileum. J. Ethnopharmacol. 2009, 122, 434-438. [CrossRef]

6. Rocchetti, G.; Lucini, L.; Ahmed, S.R.; Saber, F.R. In vitro cytotoxic activity of six Syzygium leaf extracts as related to their phenolic profiles: An untargeted UHPLC-QTOF-MS approach. Food Res. Int. 2019, 126, 108715. [CrossRef] [PubMed]

7. Rocchetti, G.; Pagnossa, J.P.; Blasi, F.; Cossignani, L.; Piccoli, R.H.; Zengin, G.; Montesano, D.; Cocconcelli, P.S.; Lucini, L. Phenolic profiling and in vitro bioactivity of Moringa oleifera leaves as affected by different extraction solvents. Food Res. Int. 2020, 127, 108712. [CrossRef] [PubMed]

8. Güneş, S.; Savran, A.; Paksoy, M.Y.; Koşar, M.; Çakılcığlu, U. Ethnopharmacological survey of medicinal plants in Karaisalı and its surrounding (Adana-Turkey). J. Herb. Med. 2017, 8, 68-75. [CrossRef]

9. Baydoun, S.; Chalak, L.; Dalleh, H.; Arnold, N. Ethnopharmacological survey of medicinal plants used in traditional medicine by the communities of Mount Hermon, Lebanon. J. Ethnopharmacol. 2015, 173, 139-156. [CrossRef]

10. Mosaddegh, M.; Naghibi, F.; Moazzeni, H.; Pirani, A.; Esmaeili, S. Ethnobotanical survey of herbal remedies traditionally used in Kohghiluyeh va Boyer Ahmad province of Iran. J. Ethnopharmacol. 2012, 141, 80-95. [CrossRef]

11. Di Giorgio, C.; Delmas, F.; Tueni, M.; Cheble, E.; Khalil, T.; Balansard, G. Alternative and Complementary Antileishmanial Treatments: Assessment of the Antileishmanial Activity of 27 Lebanese Plants, Including 11 Endemic Species. J. Altern. Complement. Med. 2008, 14, 157-162. [CrossRef] [PubMed]

12. Granato, D.; Shahidi, F.; Wrolstad, R.; Kilmartin, P.; Melton, L.D.; Hidalgo, F.J.; Miyashita, K.; van Camp, J.; Alasalvar, C.; Ismail, A.B. Antioxidant activity, total phenolics and flavonoids contents: Should we ban in vitro screening methods? Food Chem. 2018, 264, 471-475. [CrossRef] [PubMed]

13. Zengin, G.; Aktumsek, A. Investigation of antioxidant potentials of solvent extracts from different anatomical parts of Asphodeline anatolica E. Tuzlaci: An endemic plant to Turkey. Afr. J. Tradit. Complement. Altern. Med. 2014, 11, 481-488. [CrossRef] [PubMed]

14. Zengin, G.; Sieniawska, E.; Senkardes, I.; Picot-Allain, M.C.N.; Sinan, K.I.; Mahomoodally, M.F. Antioxidant abilities, key enzyme inhibitory potential and phytochemical profile of Tanacetum poteriifolium Grierson. Ind. Crops Prod. 2019, 140, 111629. [CrossRef]

15. Grochowski, D.M.; Uysal, S.; Aktumsek, A.; Granica, S.; Zengin, G.; Ceylan, R.; Locatelli, M.; Tomczyk, M. In vitro enzyme inhibitory properties, antioxidant activities, and phytochemical profile of Potentilla thuringiaca. Phytochem. Lett. 2017, 20,365-372. [CrossRef]

16. Zengin, G.; Lobine, D.; Mollica, A.; Locatelli, M.; Carradori, S.; Mahomoodally, M.F. Multiple pharmacological approaches on Fibigia eriocarpa extracts by in vitro and computational assays. Fundam. Clin. Pharmacol. 2018, 32, 400-413. [CrossRef] [PubMed]

17. El Atki, Y.; Aouam, I.; El kamari, F.; Taroq, A.; Lyoussi, B.; Taleb, M.; Abdellaoui, A. Total phenolic and flavonoid contents and antioxidant activities of extracts from Teucrium polium growing wild in Morocco. Mater. Today Proc. 2019, 13, 777-783. [CrossRef]

18. Ahmed, D.; Khan, M.M.; Saeed, R. Comparative Analysis of Phenolics, Flavonoids, and Antioxidant and Antibacterial Potential of Methanolic, Hexanic and Aqueous Extracts from Adiantum caudatum Leaves. Antioxidants 2015, 4, 394-409. [CrossRef]

19. Chávez-González, M.L.; Sepúlveda, L.; Verma, D.K.; Luna-García, H.A.; Rodríguez-Durán, L.V.; Ilina, A.; Aguilar, C.N. Conventional and Emerging Extraction Processes of Flavonoids. Processes 2020, 8, 434. [CrossRef]

20. Tang, J.; Dunshea, F.R.; Suleria, H.A. LC-ESI-QTOF/MS Characterization of Phenolic Compounds from Medicinal Plants (Hops and Juniper Berries) and Their Antioxidant Activity. Foods 2020, 9, 7. [CrossRef] [PubMed] 
21. Kumar, B.R. Application of HPLC and ESI-MS techniques in the analysis of phenolic acids and flavonoids from green leafy vegetables (GLVs). J. Pharm. Anal. 2017, 7, 349-364. [CrossRef] [PubMed]

22. Bennett, R.N.; Mellon, F.A.; Kroon, P.A. Screening crucifer seeds as sources of specific intact glucosinolates using ion-pair high-performance liquid chromatography negative ion electrospray mass spectrometry. J. Agric. Food Chem. 2004, 52, 428-438. [CrossRef] [PubMed]

23. Metlin Database. Available online: https://metlin.scripps.edu/landing_page.php?pgcontent=mainPage (accessed on 4 January 2020).

24. Linić, I.; Šamec, D.; Grúz, J.; Vujčić Bok, V.; Strnad, M.; Salopek-Sondi, B. Involvement of phenolic acids in short-term adaptation to salinity stress is species-specific among brassicaceae. Plants 2019, 8, 155. [CrossRef] [PubMed]

25. Oszmiański, J.; Kolniak-Ostek, J.; Wojdyło, A. Application of ultra performance liquid chromatography-photodiode detector-quadrupole/time of flight-mass spectrometry (UPLC-PDA-Q/TOF-MS) method for the characterization of phenolic compounds of Lepidium sativum L. sprouts. Eur. Food Res. Technol. 2013, 236, 699-706. [CrossRef]

26. Copeland, R.A.; Harpel, M.R.; Tummino, P.J. Targeting enzyme inhibitors in drug discovery. Expert Opin. Ther. Targets 2007, 11, 967-978. [CrossRef]

27. World Health Organization. Available online: https://www.who.int/news-room/fact-sheets/detail/dementia (accessed on 31 December 2019).

28. Alzheimer Association. 2019 Alzheimer's disease facts and figures. Alzheimer's Dement. 2019, 15, 321-387. [CrossRef]

29. Sabbagh, M.N.; Hendrix, S.; Harrison, J.E. FDA position statement "Early Alzheimer's disease: Developing drugs for treatment, Guidance for Industry". Alzheimer's Dement. Transl. Res. Clin. Interv. 2019, 5, 13-19. [CrossRef]

30. Li, Q.; Yang, H.; Chen, Y.; Sun, H. Recent progress in the identification of selective butyrylcholinesterase inhibitors for Alzheimer's disease. Eur. J. Med. Chem. 2017, 132, 294-309. [CrossRef]

31. Mesulam, M.M.; Guillozet, A.; Shaw, P.; Levey, A.; Duysen, E.G.; Lockridge, O. Acetylcholinesterase knockouts establish central cholinergic pathways and can use butyrylcholinesterase to hydrolyze acetylcholine. Neuroscience 2002, 110, 627-639. [CrossRef]

32. Altamura, C.; Ventriglia, M.; Martini, M.G.; Montesano, D.; Errante, Y.; Piscitelli, F.; Scrascia, F.; Quattrocchi, C.; Palazzo, P.; Seccia, S. Elevation of plasma 2-arachidonoylglycerol levels in alzheimer's disease patients as a potential protective mechanism against neurodegenerative decline. J. Alzheimer's Dis. 2015, 46, 497-506. [CrossRef]

33. Kirkan, B.; Sarikurkcu, C.; Ozer, M.S.; Cengiz, M.; Atılgan, N.; Ceylan, O.; Tepe, B. Phenolic profile, antioxidant and enzyme inhibitory potential of Onosma tauricum var. tauricum. Ind. Crops Prod. 2018, 125, 549-555. [CrossRef]

34. Sut, S.; Dall'Acqua, S.; Uysal, S.; Zengin, G.; Aktumsek, A.; Picot-Allain, C.; Mahomoodally, F. LC-MS, NMR fingerprint of Potentilla argentea and Potentilla recta extracts and their in vitro biopharmaceutical assessment. Ind. Crops Prod. 2019, 131, 125-133. [CrossRef]

35. Elufioye, T.O.; Chinaka, C.G.; Oyedeji, A.O. Antioxidant and Anticholinesterase Activities of Macrosphyra Longistyla (DC) Hiern Relevant in the Management of Alzheimer's Disease. Antioxidants 2019, 8, 400. [CrossRef] [PubMed]

36. Farràs, A.; Cásedas, G.; Les, F.; Terrado, E.M.; Mitjans, M.; López, V. Evaluation of anti-tyrosinase and antioxidant properties of four fern species for potential cosmetic applications. Forests 2019, 10, 179. [CrossRef]

37. Tlili, N.; Kirkan, B.; Sarikurkcu, C. LC-ESI-MS/MS characterization, antioxidant power and inhibitory effects on $\alpha$-amylase and tyrosinase of bioactive compounds from hulls of Amygdalus communis: The influence of the extracting solvents. Ind. Crops Prod. 2019, 128, 147-152. [CrossRef]

38. He, L.; Li, H.T.; Guo, S.W.; Liu, L.F.; Qiu, J.B.; Li, F.; Cai, B.C. Inhibitory effects of sinapine on activity of acetylcholinesterase in cerebral homogenate and blood serum of rats. Zhongguo Zhong Yao Za Zhi 2008, 33, 813-815.

39. Santos-Sánchez, N.F.; Salas-Coronado, R.; Villanueva-Cañongo, C.; Hernández-Carlos, B. Antioxidant compounds and their antioxidant mechanism. In Antioxidants; IntechOpen: London, UK, 2019.

40. Mahomoodally, M.F.; Zengin, G.; Aumeeruddy, M.Z.; Sezgin, M.; Aktumsek, A. Phytochemical profile and antioxidant properties of two Brassicaceae species: Cardaria draba subsp. draba and Descurainia sophia. Biocatal. Agric. Biotechnol. 2018, 16, 453-458. [CrossRef]

41. Wang, L.-S.; Sun, X.-D.; Cao, Y.; Wang, L.; Li, F.-J.; Wang, Y.-F. Antioxidant and pro-oxidant properties of acylated pelargonidin derivatives extracted from red radish (Raphanus sativus var. niger, Brassicaceae). Food Chem. Toxicol. 2010, 48, 2712-2718. [CrossRef] 
42. Alqahtani, F.Y.; Aleanizy, F.S.; Mahmoud, A.Z.; Farshori, N.N.; Alfaraj, R.; Al-sheddi, E.S.; Alsarra, I.A. Chemical composition and antimicrobial, antioxidant, and anti-inflammatory activities of Lepidium sativum seed oil. Saudi J. Biol. Sci. 2019, 26, 1089-1092. [CrossRef] [PubMed]

43. Chen, C. Sinapic Acid and Its Derivatives as Medicine in Oxidative Stress-Induced Diseases and Aging. Oxid. Med. Cell. Longev. 2016, 2016, 3571614. [CrossRef]

44. Khan, F.A.; Maalik, A.; Murtaza, G. Inhibitory mechanism against oxidative stress of caffeic acid. J. Food Drug Anal. 2016, 24, 695-702. [CrossRef]

45. Geran, R.; Greenberg, N.; Macdonald, M.; Schumacher, A. Protocols for screening chemical agents and natural products against animal tumors and other biological systems. Cancer Chemother. Rep. 1972, 3, 1-103.

46. Łaska, G.; Sieniawska, E.; Świątek, Ł.; Zjawiony, J.; Khan, S.; Boguszewska, A.; Stocki, M.; Angielczyk, M.; Polz-Dacewicz, M. Phytochemistry and biological activities of Polemonium caeruleum L. Phytochem. Lett. 2019, 30, 314-323. [CrossRef]

47. Srisawat, T.; Chumkaew, P.; Heed-Chim, W.; Sukpondma, Y.; Kanokwiroon, K. Phytochemical screening and cytotoxicity of crude extracts of Vatica diospyroides Symington Type LS. Trop. J. Pharm. Res. 2013, 12, 71-76. [CrossRef]

48. Thienthiti, K.; Tuchinda, P.; Wongnoppavich, A.; Anantachoke, N.; Soonthornchareonnon, N. Cytotoxic effect of compounds isolated from Goniothalamus marcanii Craib stem barks. Pharm. Sci. Asia 2017, 44, 86-95. [CrossRef]

(C) 2020 by the authors. Licensee MDPI, Basel, Switzerland. This article is an open access article distributed under the terms and conditions of the Creative Commons Attribution (CC BY) license (http://creativecommons.org/licenses/by/4.0/). 